\title{
DNA demethylation agent 5azadC downregulates HPV16 E6 expression in cervical cancer cell lines independently of TBX2 expression
}

\author{
JEROME PERRARD ${ }^{1}$, ADRIEN MOREL ${ }^{2}$, KOCEILA MEZNAD ${ }^{1}$, PHILIPPE PAGET-BAILLY ${ }^{1}$, \\ VERONIQUE DALSTEIN ${ }^{3,4}$, DAVID GUENAT ${ }^{1,5}$, CELINE MOURAREAU ${ }^{3,4}$, CHRISTINE CLAVEL $^{3,4}$, \\ SYLVIE FAUCONNET $^{1}$, AURELIE BAGUET ${ }^{1}$, CHRISTIANE MOUGIN ${ }^{1,5}$ and JEAN-LUC PRETET ${ }^{1,5}$
}

\begin{abstract}
${ }^{1}$ Équipe d'Accueil 3181, University of Bourgogne Franche-Comté, Laboratoire d'Excellence Lipoprotéines et Santé, Prévention et Traitement des Maladies Inflammatoires et du Cancer, 25000 Besançon, France; ${ }^{2}$ Center For Research in Genetics and Genomics-CIGGUR, GENIUROS Research Group, School of Medicine and Health Sciences, Universidad del Rosario, Bogotá 112041, Colombia; ${ }^{3}$ Institut National de la Santé et de la Recherche Médicale, Unité Mixte de Recherche-S 1250

Pathologies Pulmonaires et Plasticité Cellulaire, Université de Reims Champagne-Ardenne, Faculté de Médecine;

${ }^{4}$ Centre Hospitalier Universitaire Reims, Laboratoire Biopathologie, 51000 Reims; ${ }^{5}$ Centre National de Référence Papillomavirus, CHU Besançon, Boulevard Alexandre Fleming, 25000 Besançon, France
\end{abstract}

Received March 7, 2019; Accepted September 9, 2019

DOI: $10.3892 / 01.2019 .11158$

\begin{abstract}
HPV16 is the most carcinogenic human papillomavirus and causes $>50 \%$ of cervical cancers, the majority of anal cancers and $30 \%$ of oropharyngeal squamous cell carcinomas. HPV carcinogenesis relies on the continuous expression of the two main viral oncoproteins E6 and E7 that target $>150$ cellular proteins. Among them, epigenetic modifiers, including DNA Methyl Transferases (DNMT), are dysregulated, promoting an aberrant methylation pattern in HPV-positive cancer cells. It has been previously reported that the treatment of HPV-positive cervical cancer cells with DNMT inhibitor 5-aza-2'-deoxycytidine (5azadC) caused the downregulation of E6 expression due to mRNA destabilization that was mediated by miR-375. Recently, the T-box transcription factor 2 (TBX2) has been demonstrated to repress HPV LCR activity. In the current study, the role of TBX2 in E6 repression was investigated in HPV16 cervical cancer cell lines following 5azadC treatment. A decrease of E6 expression was accompanied by p53 and p21 restoration. While TBX2 mRNA was upregulated in 5azadC-treated $\mathrm{SiHa}$ and $\mathrm{Ca}$ Ski cells, TBX2 protein was not detectable. Furthermore, the overexpression of TBX2
\end{abstract}

Correspondence to: Professor Jean-Luc Prétet, Équipe d'Accueil 3181, University of Bourgogne Franche-Comté, Laboratoire d'Excellence Lipoprotéines et Santé, Prévention et Traitement des Maladies Inflammatoires et du Cancer, 19 rue Ambroise Paré, 25000 Besançon, France

E-mail: jean_luc.pretet@univ-fcomte.fr

Key words: epigenetic, DNA methyltransferase inhibitor, 5-aza-2'-deoxycytidine, human papillomavirus-induced cancer, T-Box protein in cervical cancer cells did not allow the repression of E6 expression. The TBX2 transcription factor is therefore unlikely to be associated with the repression of E6 following 5azadC treatment of SiHa and Ca Ski cells.

\section{Introduction}

High risk human papillomaviruses (HR HPV) are $\sim 55 \mathrm{~nm}$ non-enveloped circular DNA viruses causing anogenital and oropharyngeal cancers (1). Carcinogenic properties of HR HPV are mainly due to the continuous expression of E6 and E7 oncoproteins. Indeed, the most described effects of these two oncoproteins are to bind and abrogate the functions of the tumor suppressor proteins p53 and pRb respectively (2-4). This leads to altered cell cycle regulation with increased cell proliferation rate, immortalization, chromosomal instability driving malignant transformation of infected cells (5). However, E6 and E7 interact with many other proteins and particularly with epigenetic enzymes. Among them, DNMT1 is a DNA methyltransferase maintaining methylation on CpG dinucleotide during cell division. This epigenetic mark generally drives chromatin compaction, thus repressing genes under its dependence. Interestingly, E6 and E7 expression are also regulated by epigenetic mechanisms, and methylation level on viral promoter is higher in HPV-associated cancers than in precancerous lesions or in normal tissues (6). Recently, studies revealed that E6 and E7 expression was downregulated following treatment of HPV positive cancer cell lines by 5aza-2'-deoxycytidine (5azadC), a DNA methyl transferase (DNMT) inhibitor also named decitabine (7-9). The downregulation of E6 was partly due to the upregulation of miR-375 $(8,9)$, known to target the early HPV16 transcripts (10). Indeed, E6 expression was only partially restored by miR-375 inhibitor in 5azadC-treated cells (9). 
This suggests that other mechanisms are involved in E6 downregulation following 5 azadC treatment. In fact, although expression of transcription factors known to bind to early viral promoter (SP1, AP1, YY1 and NF1) is not modified by 5azadC treatment, not all transcription factor expression has been assessed. This is why the role of T-box transcription factor 2 (TBX2), another transcription factor binding viral promoter, has been evaluated.

In 2013, it has been shown that the HPV16 LCR could be repressed, at least in vitro, by $\mathrm{TBX} 2$, a member of the T-Box protein family (11). This family include transcription factors involved in embryonic development and encoded by highly conserved genes among vertebrates (12). T-Box factors recognize and bind the core sequence GGTGTGA, also known as the T-element (13). Although the HPV16 LCR lacks the canonical T-element, TBX2 is able to repress the p97 activity by binding a sequence located between $\mathrm{nt} 7564$ and $\mathrm{nt}$ 7756 (11). Conversely to other T-Box family members, TBX2 has repressive activities thanks to the presence of a strong repression domain located in its $\mathrm{C}$-terminal region.

The role of TBX2 in tumorigenesis remains controversial because of both anti- and pro-tumorigenic activities. On the one hand, TBX2 is able to bypass senescence and activate cell proliferation by repressing $\mathrm{p} 14^{\mathrm{ARF}}$ and $\mathrm{p} 21$ in different cancer models (12). TBX2 expression was significantly increased in prostate cancers compared to healthy adjacent tissues (14), suggesting an oncogenic activity. On the other hand, TBX2 inhibits cell cycle progression in lung adenocarcinoma (15) and its expression is decreased in lung cancers compared to normal tissue (16). Furthermore, TBX2 methylation has been associated with a poor prognosis in chronic lymphocytic leukemia (17), endometrial cancer (18) and bladder cancer (19). Thus, TBX2 may also exert an anti-tumor activity depending on the context and cellular origin of cancers.

In the present study, the role of TBX2 was investigated to determine whether, in addition to miR-375, it could induce E6 repression following 5azadC treatment of HPV16 cervical cancer cells.

\section{Materials and methods}

Cell lines and cell culture. MCF-7 cells [mammary cancer cells, American Type Culture Collection (ATCC)], SiHa cells (cervical cancer cells, ATCC) and U-2 OS cells (bone osteosarcoma cells, ATCC) were cultured in Dulbecco's modified Eagle's medium (DMEM); Ca Ski cells (cervical cancer cells, ATCC) were grown in Roswell Park Memorial Institute (RPMI) medium while C-33 A and HeLa cells (both cervical cancer cells, ATCC) were cultured in Eagle's minimum essential medium (EMEM). All media were supplemented with $10 \%(\mathrm{v} / \mathrm{v})$ fetal bovine serum (FBS, Lonza) but not with antibiotics. Cells were cultured at $37^{\circ} \mathrm{C}$ in a $5 \% \mathrm{CO}_{2}$ humidified incubator.

Drug treatment. SiHa and Ca Ski cells were treated by 5-aza2'-deoxycytidine (5azadC) (Epigentek). This demethylating agent was dissolved in DMSO at $220 \mathrm{mM}$ and then diluted in appropriate medium at 0.25 or $5.0 \mu \mathrm{M}$ to treat $\mathrm{SiHa}$ cells $\left(10,000\right.$ cells $\left./ \mathrm{cm}^{2}\right)$ and Ca Ski cells $\left(10,000\right.$ cells $\left./ \mathrm{cm}^{2}\right)$ for 24 , 48, 72 and $96 \mathrm{~h}$. The treatment medium was renewed every day. Untreated HeLa, C-33 A and MCF-7 cells were harvested as controls for western blotting studies.

U-2 OS, SiHa and Ca Ski cells were transfected as described below.

Plasmid transfection and luciferase assay. U-2 OS cells were plated in 96-well microplates at a density of 15,000 cells per well. They were transfected for $24 \mathrm{~h}$ with either pT-REX-DEST30/empty or pT-REx-DEST30/TBX2-3XFlag plus a mixture of pGL3-Luc-16LCR (obtained by cloning the HPV16 DNA sequence from nt 7135 to nt 105 into the pGL3 plasmid) and pRenilla (Promega) with JetPEI ${ }^{\circledR}$ transfection reagent (Polyplus-transfection) according to the manufacturer's recommendations. Cells were lysed by $1 \mathrm{X}$ Lysis reagent of Dual-Luciferase Reporter Assay System kit (Promega). The luciferase assay reagent was mixed with lysates and the luminescence was recorded using the TECAN Infinite 200 Pro instrument. Unfortunately, luciferase activity could not have been normalized by Renilla signals because TBX2 strongly repressed the CMV-driven vector pRenilla, as already documented by Schneider and collaborators (11).

$\mathrm{SiHa}$ and $\mathrm{Ca}$ Ski cells were plated in 6-well plates at a density of 350,000 cells per well and transfected with pT-REx-DEST30/empty or pT-REx-DEST30/TBX2-3XFlag using JetPEI ${ }^{\circledR}$ transfection reagent according to the manufacturer's recommendations. At $48 \mathrm{~h}$ after plasmid transfection, cells were harvested for checking overexpression efficiency and subsequent analyses via RTqPCR and western-blotting.

5azadC and TBX2 combinatory treatment. SiHa and $\mathrm{Ca}$ Ski cells were seeded at 10,000 cells $/ \mathrm{cm}^{2}$ in 6 -well plates and treated with $5 \mathrm{azadC}$ at $0.25 \mu \mathrm{M}$ for $72 \mathrm{~h}$. The treatment medium was renewed every day. Twenty-four hours after the beginning of 5azadC treatment, cells were transfected with pT-REx-DEST30/empty or pT-REx-DEST30/TBX2-3XFlag using JetPEI ${ }^{\circledR}$ transfection reagent according to the manufacturer's recommendations. Cells were then cultured for $48 \mathrm{~h}$ and harvested for E6 expression analysis by RT-qPCR.

Transfection of siRNA. MCF-7 cells were transfected with $20 \mathrm{nM}$ of siRNA targeting TBX2 (5'-GGA-GCU-GUG-GGACCA-GUU-CTT-3') or control siRNA (SR-CL000-005; Eurogentec) using Lipofectamine 2000 (Thermo Fisher Scientific, Inc.) according to the manufacturer's instructions (ratio siRNA/Lipofectamine of 1:3). Forty-eight hours after transfection, cells were harvested directly in Ribozol ${ }^{\mathrm{TM}}$ solution for RNA extraction or scrapped, centrifuged and lysed in RIPA solution for protein extraction.

RNA extraction and reverse transcription. Total cellular RNAs were isolated by RiboZol ${ }^{\mathrm{TM}}$-chloroform method (VWR). Briefly, cells were lysed in $500 \mu \mathrm{l}$ of RiboZol and $100 \mu \mathrm{l}$ of chloroform were added. After a centrifugation at $12,000 \mathrm{~g}$ $\left(15 \mathrm{~min}, 4^{\circ} \mathrm{C}\right)$, aqueous phase was harvested and incubated 10 min with $500 \mu 1$ of isopropanol. Then, total RNAs were pelleted by centrifugation at $12,000 \mathrm{~g},\left(10 \mathrm{~min}, 4^{\circ} \mathrm{C}\right)$, washed with cold ethanol and dissolved in molecular biology grade water. cDNAs were synthetized using $500 \mathrm{ng}$ of total RNA with the Maxima First Strand cDNA Synthesis kit (Thermo 
Fisher Scientific, Inc.) according to the manufacturer's recommendations.

$R T-q P C R$. Primers were synthetized by Eurogentec. Real-time quantitative PCR was performed using SYBR Green real time PCR master mix (Life Technologies) in the ABI 7500 Real-Time PCR System (Applied Biosystems). The cDNAs were amplified using the following cycling parameters: $95^{\circ} \mathrm{C}$ for $5 \mathrm{~min}$ followed by 40 cycles of $95^{\circ} \mathrm{C}$ for $30 \mathrm{sec}$ and $60^{\circ} \mathrm{C}$ for 1 min. Transcript levels for E6 and TBX2 were measured with GAGAACTGCAATGTTTCAGGACC forward and TGTATA GTTGTTTGCAGCTCTGTGC reverse primers for E6, CTC TGACAAGCACGGCTTCA forward and TGTCGTTGGCTC GCACTATG reverse primers for TBX2 and normalized with $\beta 2 \mathrm{M}$ mRNA level measured with GATGAGTATGCCGTGTG forward and CAATCCAAATGCGGCATCT reverse primers using the $2^{-\Delta \Delta \mathrm{Ct}}$ method (20).

Western blotting. Following different conditions, cells were harvested by scraping in PBS and stored in dry pellets at $-80^{\circ} \mathrm{C}$ until use. Proteins were extracted with radio immunoprecipitation assay (RIPA) lysis buffer [50 mM Tris/HCl, pH 7.4, $150 \mathrm{mM}$ $\mathrm{NaCl}, 1 \%$ (v/w) Nonidet P-40, 0.5\% (w/w) Na deoxycholate, $1 \mathrm{mM}$ EDTA, $30 \mu \mathrm{g} / \mathrm{ml}$ protease inhibitor] (Roche Diagnostics). After sonication, protein concentrations were determined using the Bio-Rad Protein assay (Bio-Rad) according to the manufacturer's recommendations. Each sample was resolved on $12 \%$ SDS-PAGE gels and then transferred to Hybond ${ }^{\circledR}$ polyvinylidene difluoride (PVDF) membranes (GE Healthcare). The membranes were blocked with $5 \%$ nonfat milk overnight at $4{ }^{\circ} \mathrm{C}$ under constant shaking and then incubated for $2 \mathrm{~h}$ at room temperature under constant agitation with primary antibodies: Anti-E6HPV16, 1/1,000 (2E-3F8; Euromedex, Souffelweyersheim, France); DO7 anti-p53, 1/2,000 (554298, BD Biosciences, Le Pont de Claix, France); 6B6 anti-p21, 1/2,000 (554228; BD Biosciences); AC15 anti- $\beta$-actin, 1/20,000 (A1978; Sigma-Aldrich); C-17 anti-TBX2, 1/500 (sc-17880). After several washes, membranes were incubated with goat anti-mouse, goat anti-rabbit (BD Pharmingen) or rabbit anti-goat (Agilent) immunoglobulin antibodies conjugated with horseradish-peroxidase. The immune complexes were revealed using an enhanced chemiluminescence detection system with Pierce ECL2 western blotting substrate (Thermo Fisher Scientific, Inc.) using ChemiDoc XRS+. The band densities were normalized against the $\beta$-actin internal control and analyzed by Image Lab software (Bio-Rad).

Statistical analysis. A two-tailed unpaired Student's t-test was used to assess differences between two groups. In each case non-treated cells served as reference control. Two-way ANOVA followed by Levene test were used to analyze differences in E6 expression following combinatory treatments. All data were obtained from at least three independent experiments or as specified for each figure and are presented as mean \pm standard deviation (SD). $\mathrm{P}<0.05$ was considered to indicate a statistically significant difference.

\section{Results}

5azadC treatment induces HPV16 E6 downregulation and p53 and p21 upregulation. As we previously published, 5azadC treatment of SiHa cells induced a time-dependent decrease of E6 expression at both mRNA and protein levels (Fig. 1A-C). The effect of 5azadC on E6 repression was observed starting $24 \mathrm{~h}$ after treatment, with a maximal decrease in transcripts reaching $60 \%$ at $96 \mathrm{~h}$ of treatment (Fig. 1C), a result consistent with the decreased expression of E6 observed at the protein level (Fig. 1A and B). In contrast, the effect of 5azadC on E6 RNA expression did not appear dependent on the concentration used $(0.25$ or $5 \mu \mathrm{M})$ to treat $\mathrm{SiHa}$ cells. At the protein levels, $5 \mathrm{azadC}$ at $5 \mu \mathrm{M}$ seemed to cause sooner and more prominent decrease in E6 expression. As expected, the decreased level of E6 was accompanied by an increased expression of p53 and an up-regulation of the cyclin dependent kinase inhibitor p21.

E6 expression was also investigated in Ca Ski cells treated with 0.25 and $5 \mu \mathrm{M}$ of $5 \mathrm{azadC}$ up to $96 \mathrm{~h}$ (Fig. 1D-F). As in SiHa cells, E6 expression was downregulated at both protein and mRNA levels. The downregulation of E6 in Ca Ski cells was also accompanied by the restoration of $\mathrm{p} 53$ and $\mathrm{p} 21$ expression.

5 azadC treatment induces TBX2 mRNA expression. A recent report has shown that TBX2 was able to bind and repress the HPV16 LCR (11). To confirm this, the pT-REX-DEST30/TBX2-3XFlag was co-transfected with pGL3-Luc-16LCR plasmid in U-2 OS cells and the p97 promoter activity was measured. As shown in Fig. 2, the overexpression of TBX2 induced a reduction of the relative luciferase activity of $40 \%$ compared to cells transfected with the empty vector. TBX2 transfection efficiency in U-2 OS cells was assessed by western blotting (Fig. S1).

Then, the expression of TBX2 was studied in 5azadC-treated $\mathrm{SiHa}$ and $\mathrm{Ca}$ Ski cells. In a first set of experiments, the specificity of TBX2 primers and anti-TBX2 antibody was confirmed in MCF-7 cells, known to express TBX2, treated by TBX2 siRNA (Fig. S1). As shown in Fig. 3A, a slight increase of TBX2 RNA level was observed in $\mathrm{SiHa}$ cells treated with $0.25 \mu \mathrm{M}$ of $5 \mathrm{azadC}$ at $96 \mathrm{~h}$. In Ca Ski cells, no variation of TBX2 expression was observed with the same treatment (Fig. 3B). In contrast, a clear increase of TBX2 RNA was observed in both cell lines for the highest concentration $(5 \mu \mathrm{M})$ of 5azadC, especially at $96 \mathrm{~h}$, reaching 3.5 and 3.8 -fold in $\mathrm{SiHa}$ and $\mathrm{Ca}$ Ski cells respectively (Fig. 3A and B). It is interesting to note that the pattern of variation of E6 mRNA (Fig. 1C and F) was not inversely related to that of TBX2.

The endogenous expression of TBX2 was then analyzed by western blotting. The TBX2 protein was not detected in $\mathrm{SiHa}$ and $\mathrm{Ca}$ Ski cells either treated or not with the highest concentration of 5azadC during 96 h (Fig. 3C). Furthermore, TBX2 was neither detected in Hela (HPV18 positive) cells and C-33 A (HPV negative) cervical cancer cell lines (Fig. 3D). In contrast, a strong signal was observed in MCF-7 cells (a positive control for TBX2 expression) or in SiHa cells transfected with pT-REx-DEST30/TBX2-3XFlag (Fig. 3C and D). It is noteworthy that TBX2 mRNA expression is 700 and 90-fold more important in MCF-7 compared to Ca Ski and SiHa cells, respectively (not shown). Thus, these data indicate that TBX2 is not expressed or at a very low level in cervical cancer cell lines compared to MCF-7 cells.

Ectopic TBX2 expression does not repress endogenous E6 expression. Then, we investigated whether ectopic TBX2 

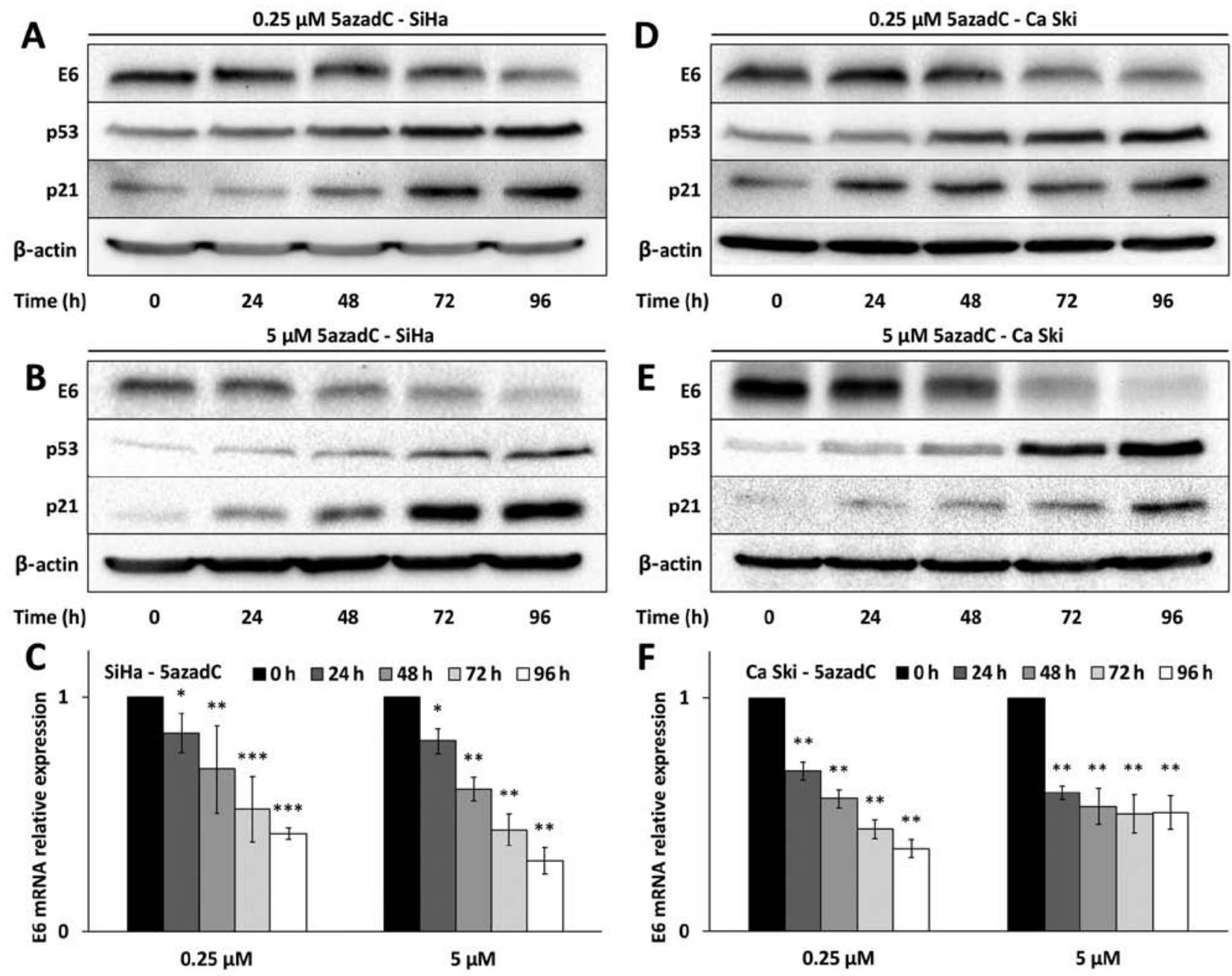

Figure 1.5azadC treatment downregulates E6 expression. E6, p53, p21 and $\beta$-actin protein expression was analyzed by western blotting in the (A) $0.25 \mu \mathrm{m}$ and (B) $0.5 \mu \mathrm{m}$ treatment in SiHa cells. (C) E6 mRNA relative expression was quantified by RT-qPCR in SiHa cells treated with 0.25 or $5 \mu \mathrm{M}$ of $5 \mathrm{azadC}$. E6, p53, p21 and $\beta$-actin protein expression was analyzed by western blotting in the (D) $0.25 \mu \mathrm{m}$ and (E) $0.5 \mu \mathrm{m}$ treatment in Ca Ski cells. (F) E6 mRNA relative expression was quantified by RT-qPCR in Ca Ski cells treated with 0.25 or $5 \mu \mathrm{M}$ of 5azadC. The data are presented as mean values from at least three independent experiments. Error bars represent the standard deviation and P-values were calculated using a Student's t-test using non-treated cells as a reference control. ${ }^{*} \mathrm{P}<0.05,{ }^{* *} \mathrm{P}<0.01$ and ${ }^{* * *} \mathrm{P}<0.001$ vs. control. 5azadC, 5-aza-2'-deoxycytidine; RT-q, reverse transcription-quantitative.

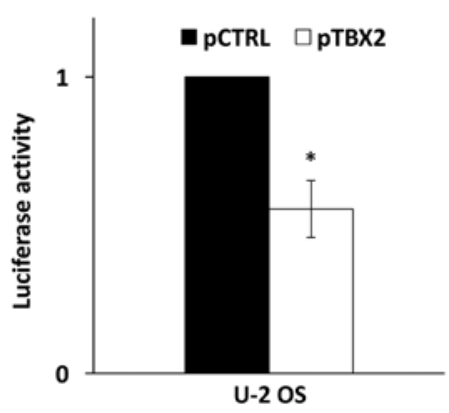

Figure 2. TBX2 ectopic expression induced relative luciferase activity repression. Luciferase activity of the reporter plasmid was measured in $\mathrm{U}-2$ OS cells transfected with the pT-REx-DEST30/TBX2-3XFlag (pTBX2) or with the control for $48 \mathrm{~h}$. The data are presented as mean values from two independent experiments. Error bars represent the standard deviation and P-values were calculated using a Student's t-test. Non-treated cells were used as a reference control: ${ }^{*} \mathrm{P}<0.05$. TBX2, T-box transcription factor 2.

expression could downregulate endogenous E6 expression in SiHa and Ca Ski cells transfected with pT-REx-DEST30/TBX23XFlag. As shown in Fig. 4A, TBX2 protein was readily detected in transfected $\mathrm{SiHa}$ and $\mathrm{Ca}$ Ski cells. But any changes were observed in E6 protein (Fig. 4A) and mRNA (Fig. 4B) expression following ectopic TBX2 expression. In keeping with this observation, TBX2 unlikely downregulates endogenous E6 expression in cervical cancer cell lines.

TBX2 overexpression and 5azadC combinatory treatment does not enhance E6 repression. In order to rule out the possibility that TBX2 was involved in E6 repression following 5 azadC treatment, TBX2 overexpression and 5azadC combinatory treatment were conducted. TBX2 overexpression has been confirmed by RT-qPCR in cells treated or not with 5azadC (Fig. S1).

Fig. 4C shows that E6 RNA relative expression was significantly decreased following 5azadC treatment in $\mathrm{SiHa}$ $(\mathrm{P}=0.03)$ and $\mathrm{Ca}$ Ski $(\mathrm{P}=0.001)$ cells. In contrast, no difference in E6 RNA relative expression was observed between the pT-REx-DEST30/TBX2-3XFlag-(pTBX2) and the pT-REx-DEST30/empty-(pCTRL) transfected cells, whether they were treated by $5 \mathrm{azadC}$ or not. Thus, overexpression of TBX2 did not enhance 5azadC-induced E6 repression. 
A

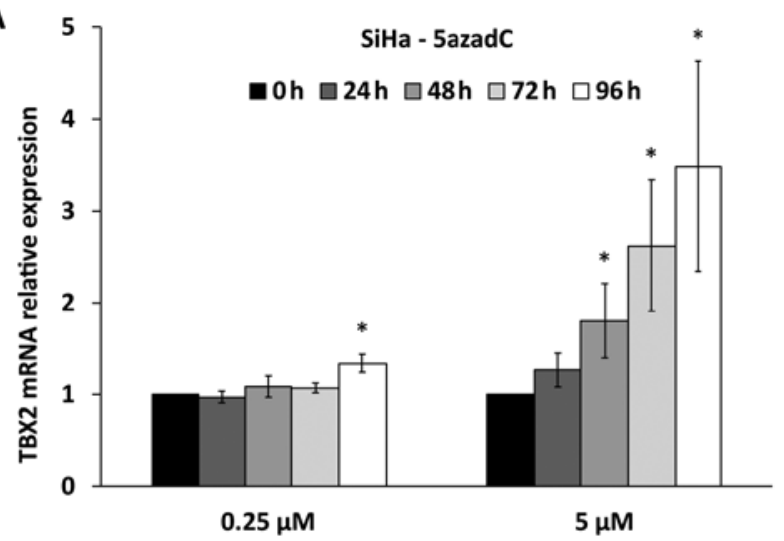

C

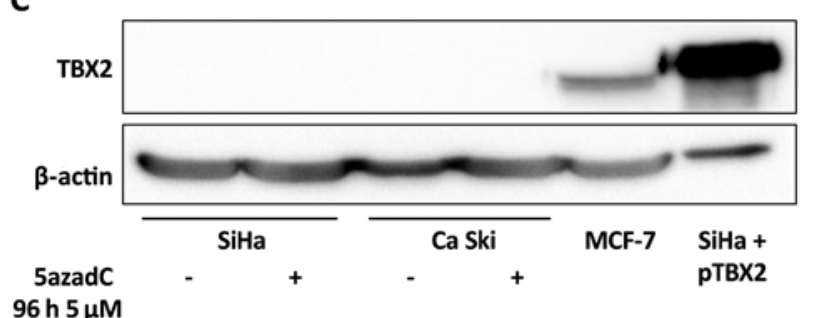

B

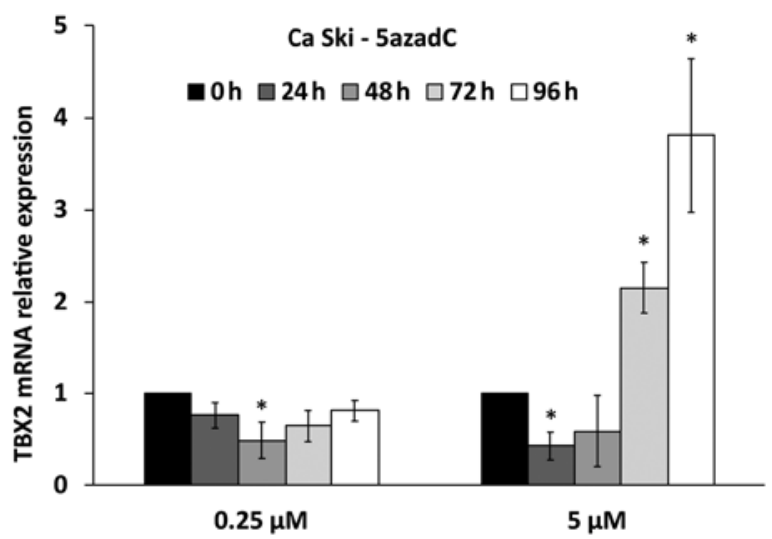

D

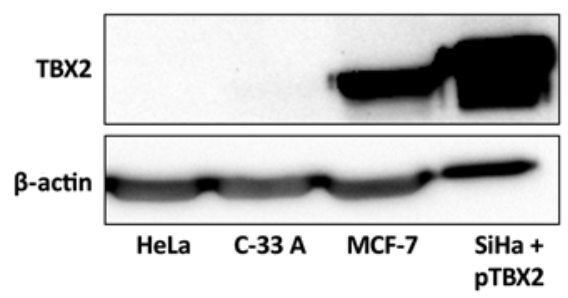

Figure 3. TBX2 mRNA detected in cervical cancer cell lines. TBX2 mRNA relative expression was analyzed using reverse-transcription-quantitative PCR in (A) SiHa cells and in (B) Ca Ski cells treated with 0.25 or $5 \mu \mathrm{M}$ of 5azadC for a period of 24, 48, 72 or 96 h. TBX2 protein expression was analyzed using western blotting in (C) SiHa and Ca Ski cells treated with $5 \mu \mathrm{M}$ of 5azadC during $96 \mathrm{~h}$ or the control and in (D) HeLa and C-33 A cervical cancer cell lines and in MCF-7 breast cancer cells or SiHa cells transfected with pT-REx-DEST30/TBX2-3XFlag (SiHa + pTBX2) that served as positive controls. The data are presented as mean values from at least three independent experiments. Error bars represent the standard deviation and P-values were calculated using a Student's t-test using non-treated cells as a reference control. ${ }^{*} \mathrm{P}<0.05$. TBX2, T-box transcription factor 2; 5azadC, 5-aza-2'-deoxycytidine.

\section{Discussion}

In this study, we confirmed that the treatment of HPV16 positive cancer cell lines with 5azadC leads to the downregulation of E6 at both mRNA and protein levels (7-9). As expected, a p53 and 21 restoration was observed in treated cells confirming the functional loss of E6. Interestingly, the treatment of the tongue HPV16-positive cancer cell line UPCI:SCC090 with $5 \mathrm{azadC}$ also leads to the decrease of E6 and to the increase of p53 and p21 expression (Fig. S2). These results, in line with those obtained by Stich et al with two other head and neck cancer-derived cell lines (UM-SCC-47 and UM-SCC-104) (8), suggest that the effect of 5azadC treatment on E6 repression is independent of the tumor origin. Since 5azadC (decitabine) is already used to treat myelodysplastic syndromes (21) and acute myeloid leukemia (22) its usefulness in the treatment of HPV-associated cancers probably deserves to be addressed. In this line, Biktasova et al recently reported that the treatment of patients presenting HPV-positive head and neck cancers with 5-azacytidine, a 5azadC analogue, induced E6 and E7 RNA repression in their tumors accompanied by reactivation of the p53 pathway and apoptosis induction (23). This is the first study presenting the potential effects of a demethylating treatment against human HPV+ tumors in a clinical trial.

In SiHa cells, the effect of 5azadC on E6 RNA repression was not dependent on the concentration used since E6 downregulation was similar whatever the concentration was $(0.25 \mu \mathrm{M}$ or $5 \mu \mathrm{M})$ at each time point over $96 \mathrm{~h}$. Similarly, Stich et al showed no dose effect of 5azadC treatment on
E6*I/E7 mRNA downregulation even with a concentration as low as $100 \mathrm{nM}$ (8). In contrast, a clear time-dependent effect of $5 \mathrm{azadC}$ treatment was observed on E6 downregulation with a maximum achieved at $96 \mathrm{~h}$. This may reflect the $5 \mathrm{azadC}$ mechanism of action that requires successive cell divisions to passively demethylate DNA leading to the progressive upregulation of factor(s) involved in direct or indirect E6 repression. However, such a time dependent effect was not reproduced in Ca Ski cells treated with $5 \mu \mathrm{M}$ of $5 \mathrm{azadC}$. At this concentration, a cut-off effect was observed starting at $24 \mathrm{~h}$ of treatment, an observation consistent with a more sensitive phenotype of Ca Ski cells regarding 5azadC effects on E6 downregulation.

In order to highlight the mechanism involved in E6 downregulation following 5azadC treatment, we explored the role of the transcription factor TBX2. Indeed, Schneider and collaborators have recently proposed that TBX2 might decrease the HPV gene expression through LCR inhibition (11). Furthermore, they mapped the minimal sequence required for TBX2 inhibition from nt 7564 to nt 7756 on the LCR. A first series of experiments permitted to confirm in an in vitro assay that the overexpression of TBX2 downregulated HPV16 LCR activity.

In both $\mathrm{SiHa}$ and $\mathrm{Ca}$ Ski cells, TBX2 mRNA expression was up-regulated following $5 \mathrm{azadC}$ exposure in a time and dose dependent manner. However, this regulation pattern differed substantially from the one observed for E6. This was especially true for the lowest concentration of 5azadC that had weak effects on TBX2 mRNA upregulation while E6 mRNA was clearly downregulated. Surprisingly, the TBX2 
A

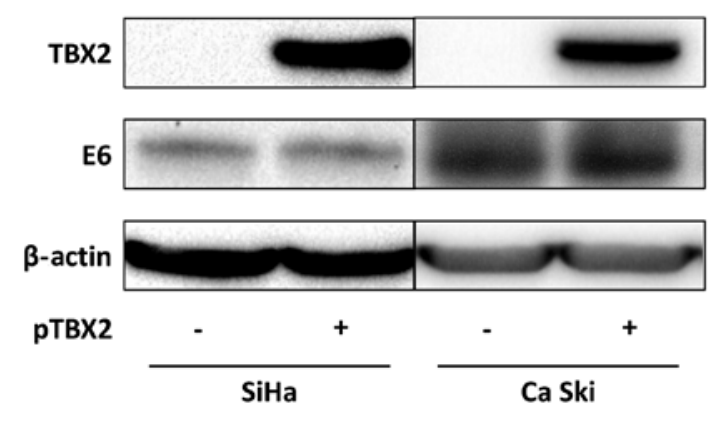

B

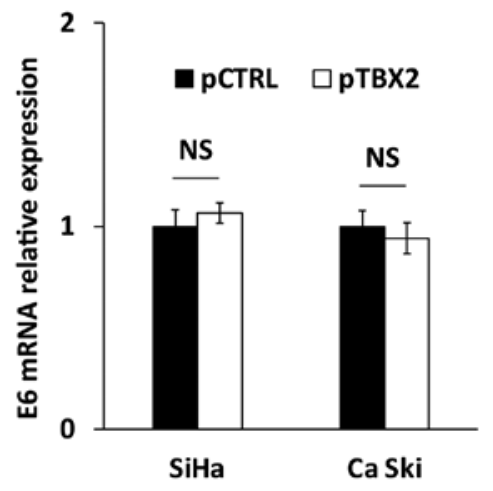

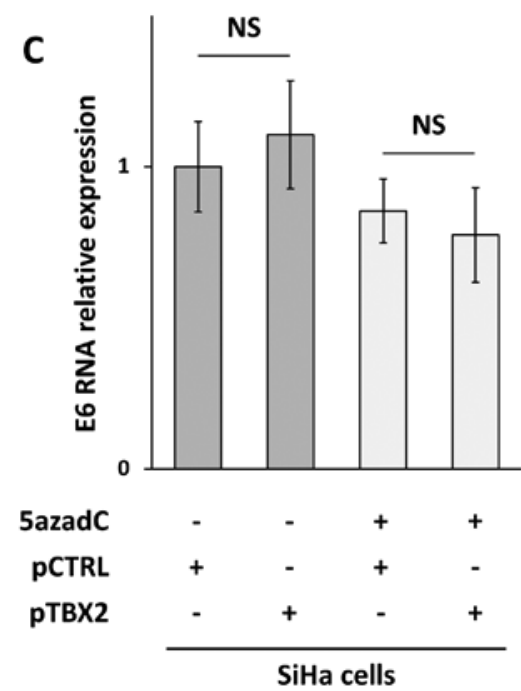

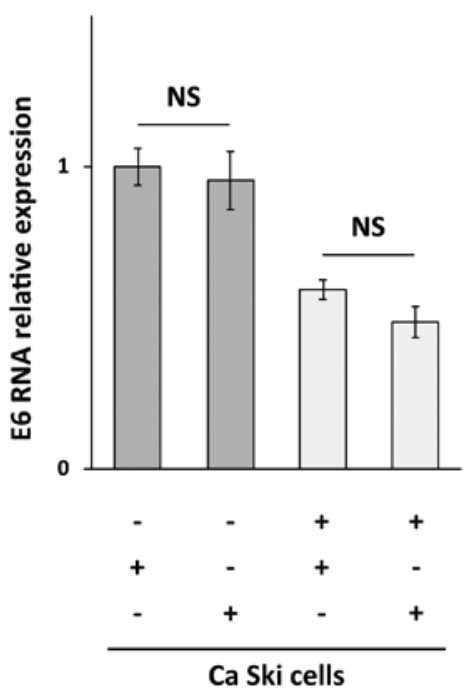

Figure 4. TBX2 is not involved in E6 repression in vivo. (A) E6 protein and (B) mRNA expression in SiHa and Ca Ski cells transfected for 48 h with pT-REx-DEST30/TBX2-3XFlag (pTBX2). The detection of TBX2 using western blotting confirmed the efficiency of transfection. E6 RNA expression in (C) $\mathrm{SiHa}$ and Ca Ski cells treated with $0.25 \mu \mathrm{M}$ of 5azadC and transfected with the pT-REx-DEST30/TBX2-3XFlag (pTBX2) or the pT-REx-DEST30/empty (pCTRL). The data are presented as mean values from three independent experiments. Error bars represent the standard deviation and P-values were calculated using a Student's t-test using non-treated cells as a reference control and a two-way ANOVA.TBX2, T-box transcription factor 2; 5azadC, 5-aza-2'-deoxycytidine; NS, not significant.

protein was undetectable in SiHa and Ca Ski cells as well as in two other cervical cancer cell lines infected by HPV18 (HeLa cells) or not infected by HPV (C-33 A cells). In contrast TBX2 protein was clearly detected in MCF-7 cells and in SiHa cells transfected with pT-REx-DEST30/TBX2-3XFlag that served as positive controls. While we can hypothesize that TBX2 protein is not expressed in cervical cancer we cannot rule out that its expression is very low in cervical cancer cells (under the limit of detection of the western blotting assay). Indeed, Schneider et al reported only a very faint band for TBX2 in their western blotting experiments performed with $\mathrm{SiHa}$ cell protein extracts. In the present study, TBX2 protein was not detected even after the treatment of cervical cancer cell lines with $5 \mu \mathrm{M}$ of $5 \mathrm{azadC}$ during $96 \mathrm{~h}$, whereas the relative expression of the corresponding mRNA was increased more than 3 -fold. Thus, we provide no evidence that the downregulation of E6 expression following 5azadC treatment is mediated through an increased expression of endogenous TBX2.

The increased expression of TBX2 mRNA transcripts without effective translation could be explained by the fact that Ca Ski and SiHa cells expressed at extremely low level TBX2 mRNA (700-fold less in Ca Ski and 90-fold less in SiHa cells), compared to TBX2 mRNA levels in MCF-7 cells as determined by RT-qPCR (data not shown). These results are in line with the Human Protein Atlas data, that report no TBX2 mRNA expression in SiHa cells (0.0 TPM) while TBX2 is expressed at 62.0 TPM in MCF-7 cells). Another explanation could be the inhibition of translation due to mRNA sequestration in the nucleus, or due to miRNA interference.

Because TBX2 protein was shown to inhibit the activity of a cloned HPV16 LCR in overexpression experiments [(11) and present study), we wondered to assess whether it could also repress the endogenous HPV16 LCR in Ca Ski and SiHa cells. While TBX2 was readily detected in both transfected cell lines, no variation in E6 expression at both protein and mRNA expression was evidenced. Similarly, TBX2 overexpression in the presence of 5azadC did not enhance E6 repression that is solely due to $5 \mathrm{azadC}$. These observations again reinforce the idea that TBX2 is unlikely involved in the regulation of E6 expression in both cell lines.

A limitation of the present study is the lack of TBX2 target assessment. No specific TBX2 target is described in cervical cancer cell models, in the literature. This is why TBX2 targets described in cell lines derived from other tissues [p21 (24), p14/p16 (25-27), p27 (28), PTEN (29) and NDRG1 (30)] were tested. However, none of these targets was significantly 
repressed following TBX2 transfection. There is no clear explanation to this observation, but it has to be noted that $\mathrm{p} 21$, p27, p14/p16 are already deregulated by HPV oncogenes in cervical cancer cells.

Epigenetic regulation of HPV16 oncogene expression is very likely since the use of a DNA demethylating agent leads to E6 mRNA and protein repression. Indirect effects linked to the re-expression of miR-375, that targets HPV16 early transcripts has been well documented $(8,9)$. By contrast, the involvement of TBX2 in E6 repression is very unlikely in $\mathrm{SiHa}$ and $\mathrm{Ca}$ Ski cells treated by $5 \mathrm{azadC}$ even if this transcription factor may exert inhibitory effect on HPV16 LCR cloned upstream a reporter gene. In particular, combinatory experiments conducted in the present study permitted to confirmed that TBX2 was not involved in E6 repression even in a context of demethylated DNA. Whether the structure of chromatin affect the accessibility of TBX2 to occupy its binding site in the native HPV16 LCR remains a challenging question.

\section{Acknowledgements}

The authors would like to thank Dr Schneider (University Medical Center of Johannes Gutenberg University Mainz, Mainz, Germany) for providing us with the pT-REx-DEST30/TBX2-3XFlag, Dr Caroline Demeret (Pasteur Institute, Paris, France) for providing us with pGL3-Luc-16LCR and Dr F Monnien (Centre Hospitalier Universitaire de Besançon, Besançon, France) for aiding in the statistical analysis of the present study. The authors would also like to thank Julie Durel and Anne Peigney (both, Université Bourgogne Franche-Comté, Université de Franche Comté, Besançon, France) for excellent technical assistance.

\section{Funding}

The present study was supported by research grants from La Ligue Contre le Cancer (grant no. CCIR-GE) and the Conseil Régional de Franche-Comté (grant no. 2016Y7570-2016Y7571). J. Perrard and A. Morel were recipients of a predoctoral scholarship from the Conseil Régional de Franche-Comté and K. Meznad was the recipient of a predoctoral scholarship from Ministère de l'Enseignement Supérieur et de la Recherche Scientifique.

\section{Availability of data and materials}

All data generated or analyzed during the present study are included in this published article.

\section{Authors' contributions}

JP, AM, ChM, VD, CC and JLP conceived and designed the experiments. JP, KM, CeM and PPB performed the experiments. JP, AB, SF, VD, DG and CC analyzed the data. JP, AB, $\mathrm{DG}, \mathrm{ChM}$ and JLP wrote the manuscript.

\section{Ethics approval and consent to participate}

Not applicable.

\section{Patient consent for publication}

Not applicable.

\section{Competing interests}

The authors declare that they have no competing interests.

\section{References}

1. Doorbar J, Egawa N, Griffin H, Kranjec C and Murakami I: Human papillomavirus molecular biology and disease association. Rev Med Virol 25 (Suppl 1): S2-S23, 2015.

2. Scheffner M, Werness BA, Huibregtse JM, Levine AJ and Howley PM: The E6 oncoprotein encoded by human papillomavirus types 16 and 18 promotes the degradation of $\mathrm{p} 53$. Cell 63: 1129-1136, 1990

3. Münger K, Scheffner M, Huibregtse JM and Howley PM: Interactions of HPV E6 and E7 oncoproteins with tumour suppressor gene products. Cancer Surv 12: 197-217, 1992.

4. Huh K, Zhou X, Hayakawa H, Cho JY, Libermann TA, Jin J, Harper JW and Munger K: Human papillomavirus type 16 E7 oncoprotein associates with the cullin 2 ubiquitin ligase complex, which contributes to degradation of the retinoblastoma tumor suppressor. J Virol 81: 9737-9747, 2007.

5. zur Hausen H: Human papillomaviruses in the pathogenesis of anogenital cancer. Virology 184: 9-13, 1991.

6. Jacquin E, Baraquin A, Ramanah R, Carcopino X, Morel A, Valmary-Degano S, Bravo IG, de Sanjosé S, Riethmuller D, Mougin C and Prétet JL: Methylation of human papillomavirus Type $16 \mathrm{CpG}$ sites at E2-binding site 1 (E2BS1), E2BS2, and the Sp1-binding site in cervical cancer samples as determined by high-resolution melting analysis-PCR. J Clin Microbiol 51: 3207-3215, 2013

7. Zhang C, Deng Z, Pan X, Uehara T, Suzuki M and Xie M: Effects of methylation status of $\mathrm{CpG}$ sites within the HPV16 long control region on HPV16-positive head and neck cancer cells. PLoS One 10: e0141245, 2015.

8. Stich M, Ganss L, Puschhof J, Prigge ES, Reuschenbach M, Guiterrez A, Vinokurova S and von Knebel Doeberitz M: 5-aza-2'-deoxycytidine (DAC) treatment downregulates the HPV E6 and E7 oncogene expression and blocks neoplastic growth of HPV-associated cancer cells. Oncotarget 8: 52104-52117, 2016.

9. Morel A, Baguet A, Perrard J, Demeret C, Jacquin E, Guenat D, Mougin C and Prétet JL: 5azadC treatment upregulates miR-375 level and represses HPV16 E6 expression. Oncotarget 8: 46163-46176, 2017.

10. Jung HM, Phillips BL and Chan EK: miR-375 activates p21 and suppresses telomerase activity by coordinately regulating HPV E6/E7, E6AP, CIP2A, and 14-3-3̧. Mol Cancer 13: 80, 2014.

11. Schneider MA, Scheffer KD, Bund T, Boukhallouk F, Lambert C, Cotarelo C, Pflugfelder GO, Florin L and Spoden GA: The transcription factors TBX2 and TBX3 interact with human papillomavirus 16 (HPV16) L2 and repress the long control region of HPVs. J Virol 87: 4461-4474, 2013.

12. Wansleben S, Peres J, Hare S, Goding CR and Prince S: T-box transcription factors in cancer biology. Biochim Biophys Acta 1846: 380-391, 2014.

13. Abrahams A, Parker MI and Prince S: The T-box transcription factor Tbx2: Its role in development and possible implication in cancer. IUBMB Life 62: 92-102, 2010.

14. Du WL, Fang Q, Chen Y, Teng JW, Xiao YS, Xie P, Jin B and Wang JQ: Effect of silencing the T-Box transcription factor TBX2 in prostate cancer PC3 and LNCaP cells. Mol Med Rep 16: 6050-6058, 2017.

15. Khalil A, Dekmak B, Boulos F, Kantrowitz J, Spira A, Fujimoto J, Kadara H, El-Hachem N and Nemer G: Transcriptomic alterations in lung adenocarcinoma unveil new mechanisms targeted by the TBX2 subfamily of tumor suppressor genes. Front Oncol 8: 482, 2018.

16. Khalil AA, Sivakumar S, Lucas FAS, McDowell T, Lang W, Tabata K, Fujimoto J, Yatabe Y, Spira A, Scheet P, et al: TBX2 subfamily suppression in lung cancer pathogenesis: A high-potential marker for early detection. Oncotarget 8: 68230-68241, 2017. 
17. Rani L, Mathur N, Gupta R, Gogia A, Kaur G, Dhanjal JK, Sundar D, Kumar L and Sharma A: Genome-wide DNA methylation profiling integrated with gene expression profiling identifiesPAX9as a novel prognostic marker in chronic lymphocytic leukemia. Clin Epigenetics 9: 57, 2017.

18. Farkas SA, Sorbe BG and Nilsson TK: Epigenetic changes as prognostic predictors in endometrial carcinomas. Epigenetics 12: 19-26, 2017.

19. Kandimalla R, van Tilborg AA, Kompier LC, Stumpel DJ, Stam RW, Bangma CH and Zwarthoff EC: Genome-wide analysis of $\mathrm{CpG}$ island methylation in bladder cancer identified TBX2, TBX3, GATA2, and ZIC4 as pTa-specific prognostic markers. Eur Urol 61: 1245-1256, 2012.

20. Livak KJ and Schmittgen TD: Analysis of relative gene expression data using real-time quantitative PCR and the 2(-Delta Delta C(T)) method. Methods 25: 402-408, 2001.

21. Gangat N, Patnaik MM and Tefferi A: Myelodysplastic syndromes: Contemporary review and how we treat. Am J Hematol 91: 76-89, 2016

22. Nieto M, Demolis P, Béhanzin E, Moreau A, Hudson I, Flores B Stemplewski H, Salmonson T, Gisselbrecht C, Bowen D and Pignatti F: The european medicines agency review of decitabine (Dacogen) for the treatment of adult patients with acute myeloid leukemia: Summary of the scientific assessment of the committee for medicinal products for human use. Oncologist 21: 692-700, 2016.

23. Biktasova A, Hajek M, Sewell A, Gary C, Bellinger G Deshpande HA, Bhatia A, Burtness B, Judson B, Mehra S, et al: Demethylation therapy as a targeted treatment for human papillomavirus-associated head and neck cancer. Clin Cancer Res 23 7276-7287, 2017
24. Prince S, Carreira S, Vance KW, Abrahams A and Goding CR: Tbx2 directly represses the expression of the p21(WAF1) cyclin-dependent kinase inhibitor. Cancer Res 64: 1669-1674, 2004

25. Jacobs JJ, Keblusek P, Robanus-Maandag E, Kristel P, Lingbeek M, Nederlof PM, van Welsem T, van de Vijver MJ, Koh EY, Daley GQ and van Lohuizen M: Senescence bypass screen identifies TBX2, which represses Cdkn2a (p19(ARF)) and is amplified in a subset of human breast cancers. Nat Genet 26 : 291-299, 2000

26. Vance KW, Carreira S, Brosch G and Goding CR: Tbx2 is overexpressed and plays an important role in maintaining proliferation and suppression of senescence in melanomas. Cancer Res 65: 2260-2268, 2005.

27. Harrelson Z, Kelly RG, Goldin SN, Gibson-Brown JJ, Bollag RJ, Silver LM and Papaioannou VE: Tbx2 is essential for patterning the atrioventricular canal and for morphogenesis of the outflow tract during heart development. Development 131: 5041-5052, 2004.

28. Lüdtke TH, Rudat C, Wojahn I, Weiss AC, Kleppa MJ, Kurz J, Farin HF, Moon A, Christoffels VM and Kispert A: Tbx2 and Tbx3 act downstream of shh to maintain canonical wnt signaling during branching morphogenesis of the murine lung. Dev Cell 39: 239-253, 2016.

29. Zhu B, Zhang M, Williams EM, Keller C, Mansoor A and Davie JK: TBX2 represses PTEN in rhabdomyosarcoma and skeletal muscle. Oncogene 35: 4212-4224, 2016.

30. Redmond KL, Crawford NT, Farmer H, D'Costa ZC, O'Brien GJ, Buckley NE, Kennedy RD, Johnston PG, Harkin DP and Mullan PB: T-box 2 represses NDRG1 through an EGR1-dependent mechanism to drive the proliferation of breast cancer cells. Oncogene 29: 3252-3262, 2010. 